\title{
Touch Input on Curved Surfaces
}

\author{
Anne Roudaut, Henning Pohl, and Patrick Baudisch \\ Hasso Plattner Institute, Potsdam, Germany \\ \{anne.roudaut, henning.pohl, patrick.baudisch\}@hpi.uni-potsdam.de
}

\begin{abstract}
Advances in sensing technology are currently bringing touch input to non-planar surfaces, ranging from spherical touch screens to prototypes the size and shape of a pingpong ball. To help interface designers create usable interfaces on such devices, we determine how touch surface curvature affects targeting. We present a user study in which participants acquired targets on surfaces of different curvature and at locations of different slope. We find that surface convexity increases pointing accuracy, and in particular reduces the offset between the input point perceived by users and the input point sensed by the device. Concave surfaces, in contrast, are subject to larger error offsets. This is likely caused by how concave surfaces hug the user's finger, thus resulting in a larger contact area. The effect of slope on targeting, in contrast, is unexpected at first sight. Some targets located downhill from the user's perspective are subject to error offsets in the opposite direction from all others. This appears to be caused by participants acquiring these targets using a different finger posture that lets them monitor the position of their fingers more effectively.
\end{abstract}

ACM Classification: H5.2 [Information interfaces and presentation]: User Interfaces: Input Devices and Strategies, Interaction Styles.

Keywords: touch, non-planar, targeting, curved, flexible, pointing, shape of device, industrial design, form factor.

General terms: Human factors.

\section{INTRODUCTION}

Recent advances in sensor technology have allowed touchenabling non-planar surfaces. Examples include capacitive sensors in Rekimoto's Smart Skin [17] and in Apple's Magic Mouse, resistive sensors in the UnMousePad [18], and FTIR-based sensing in Mouse 2.0 [24]. We also have started to see non-planar touch screens, such as Sphere [3].

For large touch surfaces, such as Sphere, surface curvature is comparably small. The smaller the device, however, the stronger the average curvature becomes, as illustrated by Figure 2. The surface of the DI-based Mouse 2.0 corresponds to a $\varnothing 15 \mathrm{~cm}$ sphere and by sensing touch through an optical fiber bundle, FlyEye [29] manages to touch-enable a $\varnothing 4 \mathrm{~cm}$ ping-pong ball. As sensing technology continues

Permission to make digital or hard copies of all or part of this work for personal or classroom use is granted without fee provided that copies are not made or distributed for profit or commercial advantage and that copies bear this notice and the full citation on the first page. To copy otherwise, or republish, to post on servers or to redistribute to lists, requires prior specific permission and/or a fee.

CHI 2011, May 7-12, 2011, Vancouver, BC, Canada.

Copyright 2011 ACM 978-1-4503-0267-8/11/05...\$10.00. to evolve, it seems plausible that even smaller devices, such as watches or even electronic jewelry, might become touch sensitive in the near future, resulting in touch surfaces of extreme curvature.

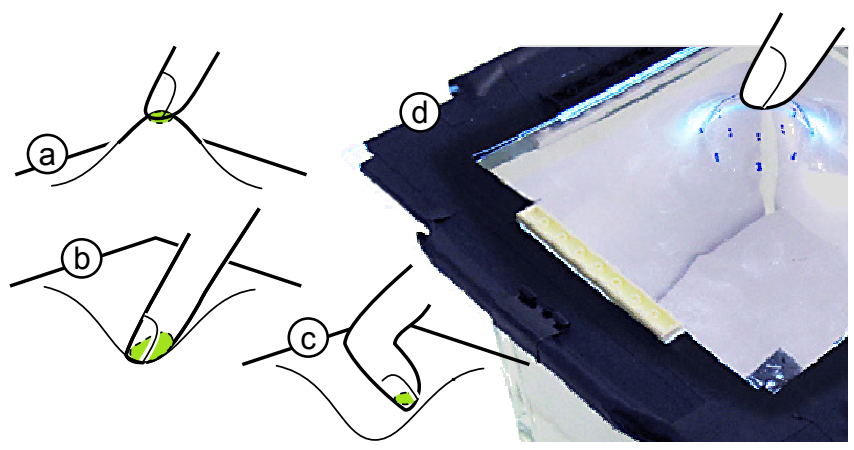

Figure 1: Summary of findings: (a) Surface convexity increases pointing accuracy and (b) concave surfaces are subject to larger error offsets. This is likely caused by how concave surfaces hug the user's finger thus resulting in a larger contact area. (c) When acquiring targets on a downhill slope participants employ a hooked finger gesture, which helps them target more effectively. (d) The FTIR-based prototype we used in our studies.

As researchers and engineers create these future touch devices, the question arises of how to design usable interfaces for them. Unfortunately, there is no empirical data about the human factors of touch on curved surfaces yet.

On flat surfaces, touch is comparably well understood. In particular, there is a series of studies investigating the factors responsible for the inaccuracy of touch, including the fat finger problem [26] and the (generalized) perceived input point model $[26,13]$. While this paper is only a first step, our ultimate goal is to create similar metric for the usability of object surfaces of arbitrary shape and curvature. Such a metric would allow industrial designers to assess the usability of devices, similar to how the measurement of wind resistance has brought rigor to the design of the shape of cars.

Touch on arbitrary shapes is of very high dimensionality, because device, hands, and the way they can make contact are all of very high degree of freedom. As a first step, we select a tractable, self-contained subset of variables, namely, single touch on spherical shapes, as these already fit existing devices.

We present a user study in which participants acquired targets on surfaces of different curvature and at locations of different slope. We report how surface curvature affects pointing accuracy (preview in Figure 1). We provide minimum button sizes to help interface designers find the best 
location for their controls on a curved surface. We also report systematic error offsets that allow engineers to increase the accuracy of their devices by compensating for them [13].

\section{RELATED WORK}

The work presented in this paper is related to non-planar touch-sensitive objects and to research on touch input.

\section{Non-planar touch devices}

Curved touch devices include relative pointing devices, such as the aforementioned Mouse 2.0 [24], and absolute pointing devices/touch screens, such as Sphere [3]. Devices can be touch-enabled using a range of technologies, such as capacitive (e.g., Smart skin [17]), resistive UnMousePad [18], and optical (e.g., FTIR [9, 24]). Many other sensor concepts could be adapted to non-planar surfaces, such as GelForce, a device that extracts directional pressure from touch [25].

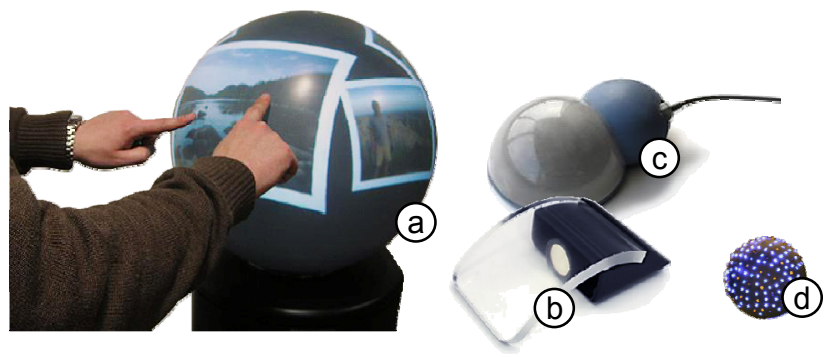

Figure 2: Selection of curved touch devices by decreasing curvature: (a) Microsoft Sphere, (b) FTIR-based and (c) DIbased Mouse 2.0, and (d) FlyEye (not to scale).

In addition to the rigid devices mentioned earlier, curved surfaces also occur as a side effect of deformable devices, including Organic User Interfaces, such as Paper Windows [12]. Objects may either be deformed by users, such as the optically sensed PhotoelasticTouch [19], Gummi [20], or even human skin (Skinput [11]), or objects may be deformed using a device, such as inflatable buttons [10].

While the majority of non-planar devices are still inputonly, we are starting to see the first non-planar or deformable touch screens, to date primarily using projection [12].

Touch is related to (but different from) grasping, which is touch with the support by an opposing thumb. Examples of graspable interfaces include Bar of Soap [23] and FlyEye [29].

\section{Understanding pointing and touch input}

Modeling target acquisition has a long tradition. Fitts' Law models targeting time for one-dimensional targets [6]. Grossman and Balakrishnan's probabilistic pointing models two-dimensional target acquisition [8].

Touch screens were initially considered inherently inaccurate because of the softness of human fingertips and the occlusion of the target by the finger (fat finger problem [26]). Touch screens were, however, adapted to highprecision pointing using localized $\mathrm{CD}$ ratio adjustments (high-precision touch screen [21]) and extensions that pre- vent the user's finger from occluding the target (e.g., offset cursor [16], shift [26]).

While touch systems traditionally reduce contact areas to points [27], more recently researchers have proposed considering the entire contact area as input, such as Shapetouch [4] and Sliding Widgets [15].

\section{Touch and angles between finger and surface}

Several researchers have found systematic effects that cause a touch device to sense touch at an offset from the intended target. The Shift technique includes a corrective offset that compensates for differences between target location and the perceived input point [26]. Benko et al. noticed that the center of the contact area moves under pressure [2]. Forlines et al. found that touching a target using a flat finger angle leads to an offset input location [7]. Wang and Ren found that finger posture and motion impact the size of the contact area [27]. Holz and Baudisch found that differences in finger roll as well as differences in users' mental models result in additional offsets. They generalized the concept of offsets into the generalized perceived input point model [13]. Follow-up work by the same authors [14] explains error offsets as a conceptual mismatch between users and devices: users target by placing a fixed point located on top of their fingernail over the target. Touch devices, in contrast, determine the contact point as the center of the contact area between finger and device.

\section{Measuring touch targeting error as offset + spread}

Because of the presence of systematic offsets, researchers have started to specify touch inaccuracy using two variables, i.e., offset and spread [13] (also referred to as constant and variable error [5)). Since we use this metric to report our results, we discuss it in additional detail.

Each targeting interaction produces a contact point, generally computed as the center of gravity of all points in the contact area, e.g., the center of an oval fitted to the contact area (Figure 3a). All contact points together can now be summarized using two variables:

Error offset refers to the distance between the centroid of a cluster of contact points and the target, measured in millimeters (Figure 3c). Offsets can be compensated for by applying corrective offsets, which is a method for increasing the accuracy of a touch device. Offsets are therefore particularly relevant for device designers.
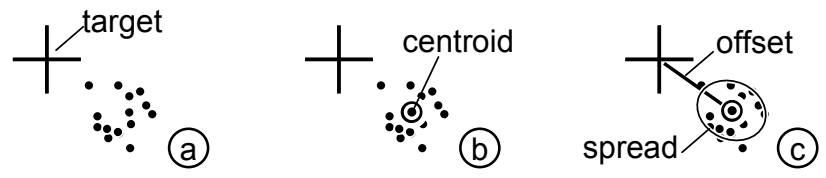

Figure 3: We report targeting error as offset and spread. (a) A series of trials results in contact points. (b) Contact points are aggregated into a centroid. (c) Offset is defines as the distance between the centroid and the target; spread is the size of the smallest button to contain $95 \%$ of all contact points.

Error spread is the remaining error after error offsets have been compensated for (Figure 3c). Spread is measured as a 
minimum button size, i.e., the diameter of the smallest circular button in millimeters that still contains $95 \%$ of all target acquisitions [13]. Note that this assumed button is centered on the centroid, not the target.

The findings in this paper allow us to extend this reasoning about offset and spread to curved surfaces.

\section{GENERALIZING FROM FLAT TO CURVED SURFACES}

In this section, we attempt to generalize what we know about touch on flat surfaces to curved surfaces. We use this to derive the hypotheses for our user study.

On flat surfaces, a finger of given posture always makes contact with the surface the same way. When we generalize to curved surfaces, the curvature of the surface affects the shape and size of the contact area. As illustrated by Figure 4 , convex surfaces curve away from the finger, resulting in a smaller contact area. Concave surfaces, in contrast, hug the finger, which leads to a larger contact area.

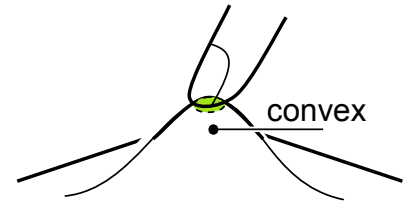

(a)

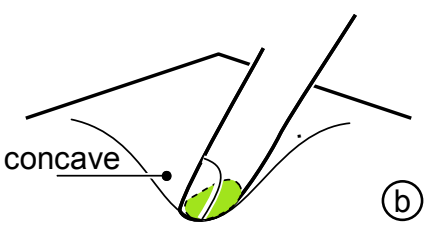

(b)
Figure 4: The contact area between finger and device (a) increases for concave and (b) decreased for convex surfaces.

In addition, the individual patches of a curved surface have different slopes, which causes the finger to make contact with the surface at different angles.

Our initial hypothesis was that users would maintain a constant finger posture, as shown in Figure 5a. For downhill slopes (from the user's perspective) this would have caused their fingers to form a flatter angle with the surface, yielding a larger contact area between finger and surface, and thus would have potentially caused larger offsets.
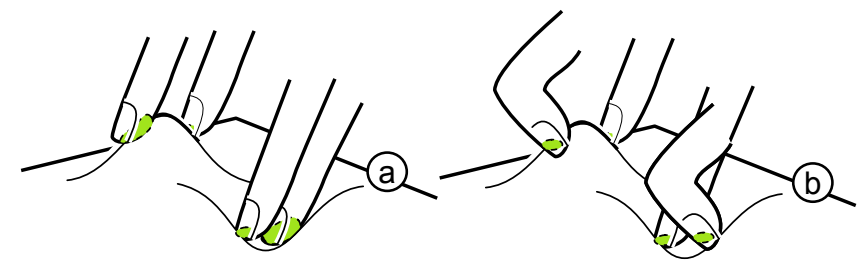

Figure 5: (a) Our initial hypothesis was that users would acquire all targets sing the same finger posture. (b) Piloting, however, revealed that most participants target downhill slopes using a hooked finger.

During piloting, however, we found that the finger contact area was largely unchanged across downhill and uphill facing slopes. Closer inspection revealed that our assumption about the finger posture was wrong. Instead, participants had targeted on downhill slopes with a hooked finger, as illustrated by Figure 5b. This posture allowed participants to hit the target surface at a roughly constant angle, which helped them minimize the contact area between their finger and the touch surface.

\section{Hypotheses}

Given that larger contact areas correlated with larger offsets on flat surfaces [13], we hypothesized that the same holds for curved surfaces. Because of the finger hugging property of concave surfaces we hypothesize

H1: Concavity increases offsets, convexity reduces it

For the same reasons we hypothesized

\section{H2. Concavity increases spread, convexity reduces it}

The observed variations in finger postures prevented us from formulating a clear hypothesis on surface slopesince flat surfaces offer nothing to reach around, hookshaped finger postures had not been studied here. Which posture would lead to better targeting was hard to predict. Consequently, rather than formulating a hypothesis we decided to

\section{Q1: Explore effect of uphill/downhill slope on offset \\ Q2: Explore effect of uphill/downhill slope on spread}

\section{CURVE TOUCH: STUDY PROTOTYPE BASED ON FTIR}

To be able to analyze the impact of the factors discussed above, we needed a device that could observe the exact contact area between the finger and the touch surface in high resolution. Since diffuse illumination (e.g., [10]) delivers only vague contour data, and capacitive sensing (such as Smart Skin [17]) is hard to manufacture for high and non-interpolated resolution, we opted for a custom design based on FTIR [9], technology previously used, for example, in Mouse 2.0 [24]. FTIR offers high resolution, a comparably crisp contact area outline, as well as reliable recognition of contact. On the flipside, FTIR starts bleeding out light with increasing curvature, which required us to make a series of modifications.

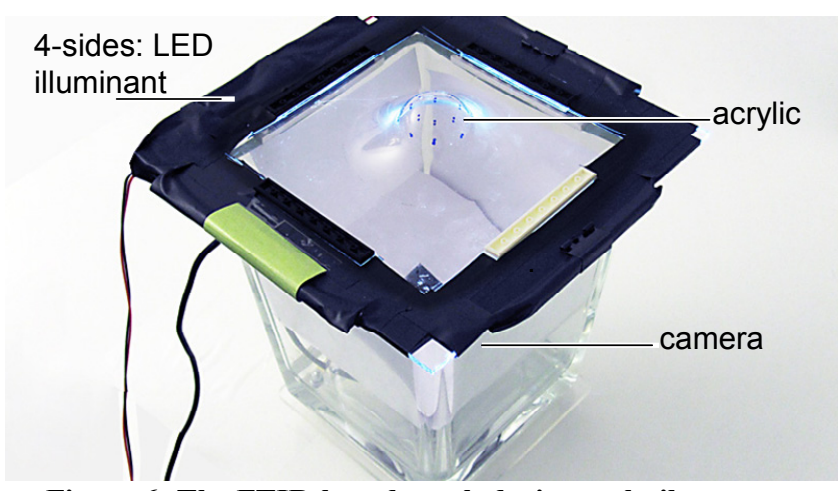

Figure 6: The FTIR-based touch device we built to sense touch on curved surfaces (curve touch).

Figure 6 shows our prototype device which we call curve touch. The basic FTIR design consists of the three familiar elements: (1) an acrylic touch surface, (2) a set of 8 bright white LEDs on each of four sides that inject light into the acrylic and (3) a high-definition web camera that observes the touch surface from below. As for all FTIR devices, a finger touching the surface causes the LED light to escape at the contact area, which is observed by the camera. We used a MS Lifecam (720p HD sensor, $30 \mathrm{fps}$ ). We processed the resulting image using OpenCV/Emgu.Cv. 


\section{Creating exact curvatures by stamping}

To obtain exact surface curvatures, we deformed the acrylic using a series of stamps, as illustrated by Figure 7 . We heated the acrylic locally using a heat gun (Figure 7a). Once malleable, we stamped shapes of the desired curvature into the acrylic (Figure $7 \mathrm{~b}$ ). Resting the acrylic on a larger ring allowed us to create the hemispherical target surface as well as a smoother transition to its periphery, which helped reduce light leakage (see next section). We obtained best results using $3 \mathrm{~mm}$ acrylic sheets, which are thin enough to allow for easy deformation, yet still thick enough to allow for the injection of light.
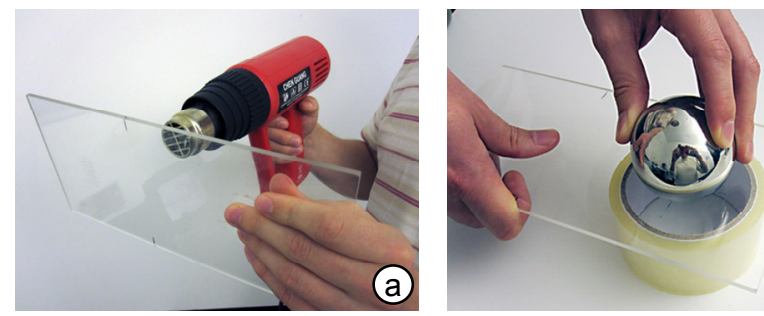

(b)

Figure 7: We created curved touch surfaces by (a) heating up the acrylic touch surface using a heat gun and (b) stamping curved objects (e.g. a silver sphere) into it.

Figure 8 shows six of the stamps we have experimented with. The four sizes we used in our user studies are highlighted in bold face.

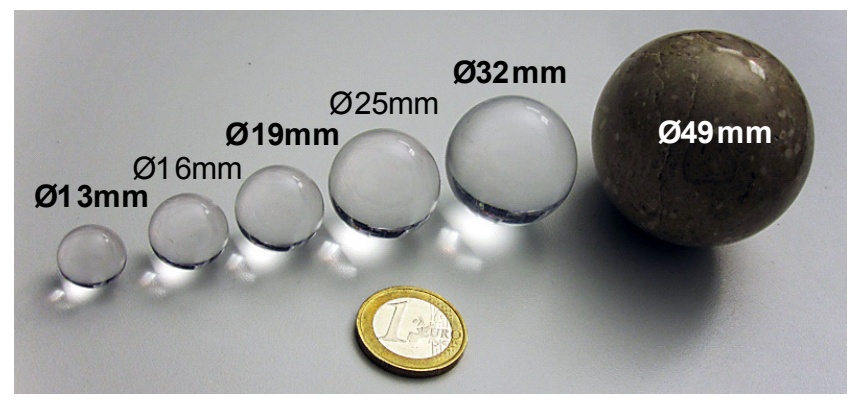

Figure 8: The stamps we used to make curved surfaces.

\section{Design modifications for the curved touch surface}

The necessity of distinguishing the light resulting from touch from other light sources required us to make some modifications:

1. No compliant surface: FTIR is most commonly used with a compliant surface layer to increase the frustration of light on contact. Unfortunately, the strong curvature of some of the shapes we used made it difficult to obtain accurately fitting compliant surfaces. We consequently dropped the compliant surface from our design. Instead, we used silicone spray to increase frustration when necessary.

2. Light leakage: Light leakage is inherent to all waveguides and only depends on curvature. It was not an issue at the actual bulge because the remaining light was strong enough and because we eliminated brightness differences by thresholding. At the edge of the bulge, it manifested as hotspots in the camera image (Figure 9b), because light was reflected off the opposite side of the bulge and into the camera as illustrated by Figure 9a. Smoothing the transition between bulge and periphery reduced the problem far enough that we could suppress it using thresholding.
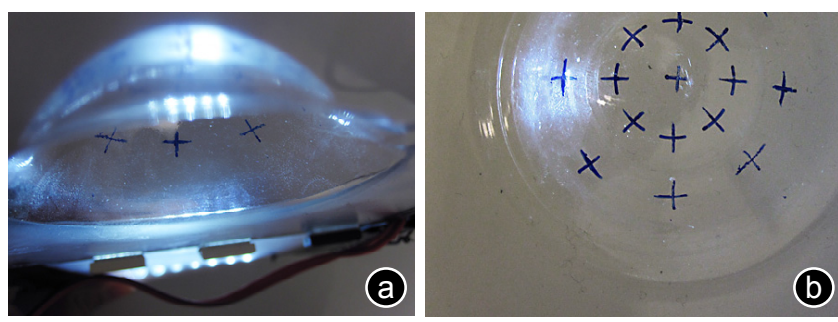

Figure 9: (a) Light injected from behind this bulge leaks at the transition from flat surface to bulge. (b) The same scene as seen by the built-in camera. The light injected from the right is reflected off the bulge and shows up as a hotspot on the left.

\section{Exchangeable touch surfaces}

To support multiple curvatures, we created different top units, each of which consisted of a differently deformed acrylic sheet with illumination (Figure 10). Snap connectors made from Lego bricks assured precise positioning of the top unit yet allowed replacing top units quickly. We also added a flat top unit to obtain a total of nine surfaces: a flat unit plus four curved units that could be flipped to serve as convex or concave shapes.

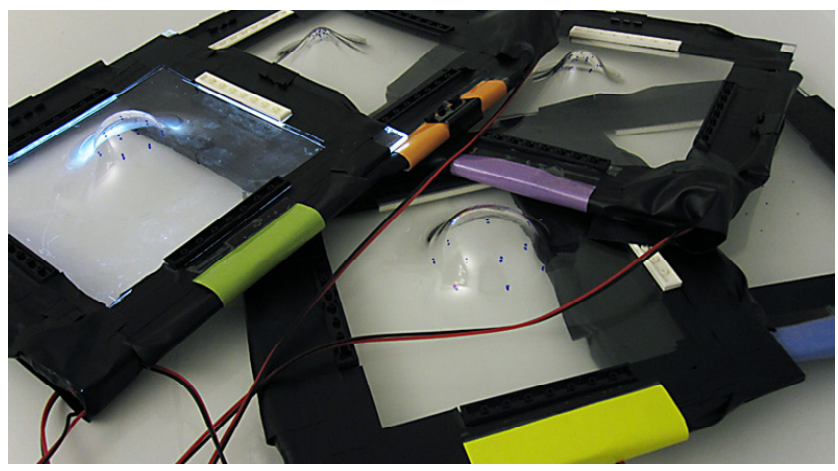

Figure 10: We implement different curvatures by using replaceable top units.

\section{Compensating for optical distortion}

The small fixed-focus lens offers a high depth of field, thus a clear image for all shapes. However, perspective effects make the curvature of the touch surfaces appear distorted. In particular, surface patches on convex bulges appear larger, because they are located further away from the camera; in addition, tilted surface patches appear deformed, because of foreshortening. A universally applicable correction for this distortion would require switching to a $3 \mathrm{D}$ representation of the surface.

Since we were only concerned with the relative position of contact points with respect to the target, however, we treated the respective patch of surface as if it were flat, which allowed us to scale with a simple linear transformation. We first restored the apparent size of the respective patch by scaling it proportional to its distance to the camera lens. We then stretched points by scaling them with the corresponding patch ratio. 


\section{USER STUDY: IMPACT OF CURVATURE ON ACCURACY}

In this study, we investigated the impact of target curvature on touch accuracy. Participants acquired targets on the curve touch. Using different top units, we varied curvature in nine levels from convex to flat to concave. By using multiple targets placed across the curved surface we also varied slope. Our goal was to test the hypotheses discussed earlier, i.e., to determine how curvature and slope impact offsets and spread.

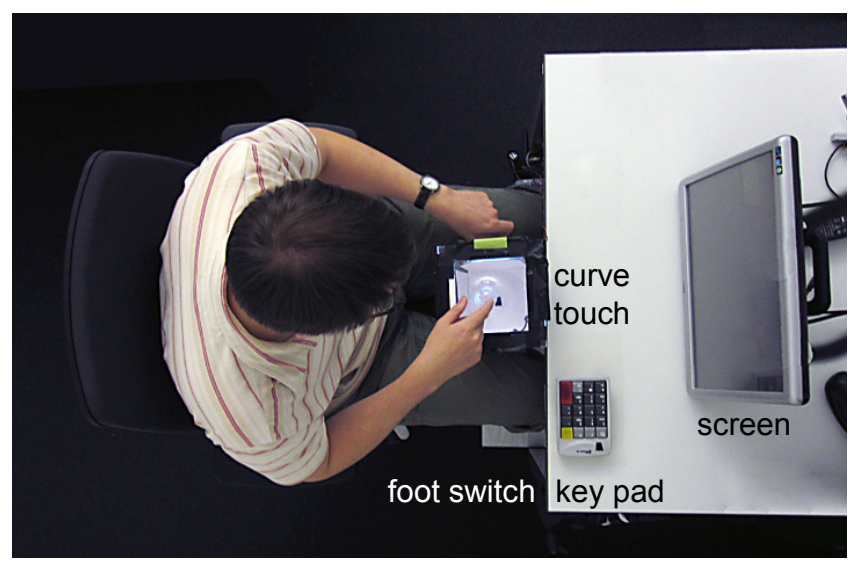

Figure 11: Apparatus: participant acquiring a target on the curve touch, here with a convex top unit.

\section{Interface}

Figure 11 shows the apparatus, consisting of the curve touch device, a screen presenting instructions, a numeric keypad for starting trials, and a foot switch for committing trials. To compensate for the depth of the curve touch device, it was mounted on a tripod, bringing its touch surface to the same height as the table. The screen was located $50 \mathrm{~cm}$ behind the curve touch and the keypad $30 \mathrm{~cm}$ behind and right of it. The curve touch device, screen, keypad, and foot switch were driven by a PC running Windows Vista.

\section{Task}

For each trial, participants were presented with a diagram illustrating the target to acquire (Figure 12a). Participants then pressed the enter key that was highlighted using red tape on the numeric keypad (Figure 12b) with their right hands and committed by pressing the foot switch (Figure 12c). We assured that all participants were seated so as to reach the device at a $45^{\circ}$ angle as shown in Figure 11.

Participants then acquired the target on the curved surface with the same (right) hand (Figure 12d) and again committed by pressing the footswitch (Figure 12e). This completed the trial and played a sound.

When participants activated the footswitch twice, i.e. before a touch, the system discarded the input and played a sound. Participants then had to repeat the trial. Errors were rare in the study $(<10 /$ participant $)$.

As common in this type of study [13], participants did not receive feedback about the touch location registered by the device. This ensured that the participants acquired the target based on their own mental model of touch, rather than being trained by the device during the study.
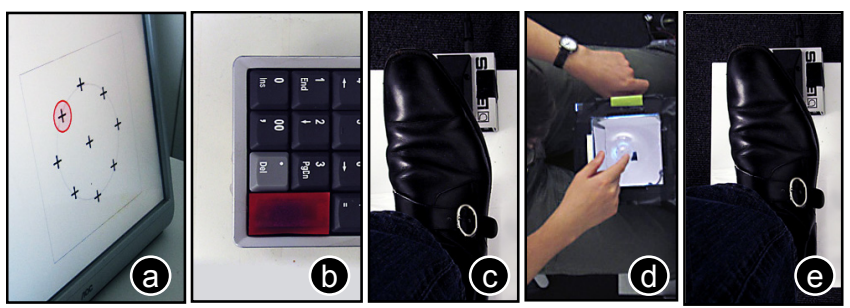

Figure 12: (a) The screen showed which target to acquire. (b) Participants pressed the start button and (c) committed using the footswitch. (d) Then they acquired the target with the same hand and (e) again committed using the footswitch.

We took the following three measures to minimize the impact of other potential factors. First, participants kept their heads in a fixed position above the touchpad, as shown in Figure 11, which controlled for parallax. Second, the use of a footswitch allowed us to avoid artifacts common with other commit methods, such as inadvertent motion during take-off. The unified button and target acquisition using the footswitch helped reduce participants' cognitive load. Finally, participants were told to focus on accuracy not on speed; consequently, we did not record task completion time.

\section{Independent Variables: Curvature and Slope}

Curvatures were implemented using the five top units (see in Figure 10). We varied slope by using targets at different locations on the curved surface. 8 targets were organized in a ring located at $45^{\circ}$ zenith angle for each curved surface; in addition there was a single target at the apex.

To prevent participants from (unintentionally) biasing their targeting towards open space we added a second ring of unused/fake crosses further outside. In addition, participants were told that there was no penalty for getting close to other targets during targeting. Note that there was no reason to include real distracter targets though. Distracters have a major effect on adaptive input techniques, such as magnetic targets (e.g., [1]), but not on unmodified touch.

\section{Experimental design}

The study used a $9 \times 9$ within-participant design, with independent variables curvature (convex or concave $\varnothing 13 \mathrm{~mm}$, $\varnothing 19 \mathrm{~mm}, \varnothing 32 \mathrm{~mm}$ and flat) and slopes (i.e. 8 targets in a ring at $45^{\circ}$ zenith plus apex). Participants performed 6 trials for each curvature.

Curvature was counterbalanced within participants using a partial Latin Square design. The order of targets was randomized. Each participant completed all conditions: 9 curvatures $\times 9$ target orientations $\times 6$ trials $=486$ trials per participant.

Participants performed 5 minutes of training before the experiment. They were allowed to take breaks every 54 selections. They completed the study in 45 minutes or less.

\section{Participants}

We recruited 12 right-handed participants (2 female) from our institution. They were between 20 and 32 years old. They received a small compensation for their time, and we awarded $€ 20$ to the most accurate participant. 


\section{Hypotheses}

Our goal was to investigate our 4 hypotheses and questions:

H1: Concavity increases offsets, convexity reduces it

H2. Concavity increases spread, convexity reduces it

Q1: Explore effect of uphill/downhill slope on offset

Q2: Explore effect of uphill downhill slope on spread

In addition, we wanted to verify that this basic observation for flat surfaces continues to hold true on curved surfaces: HO: Offsets are oriented along the user's finger

\section{Results}

Figure 13 shows the resulting raw data, i.e., all contact points by all participants as recorded during the study.

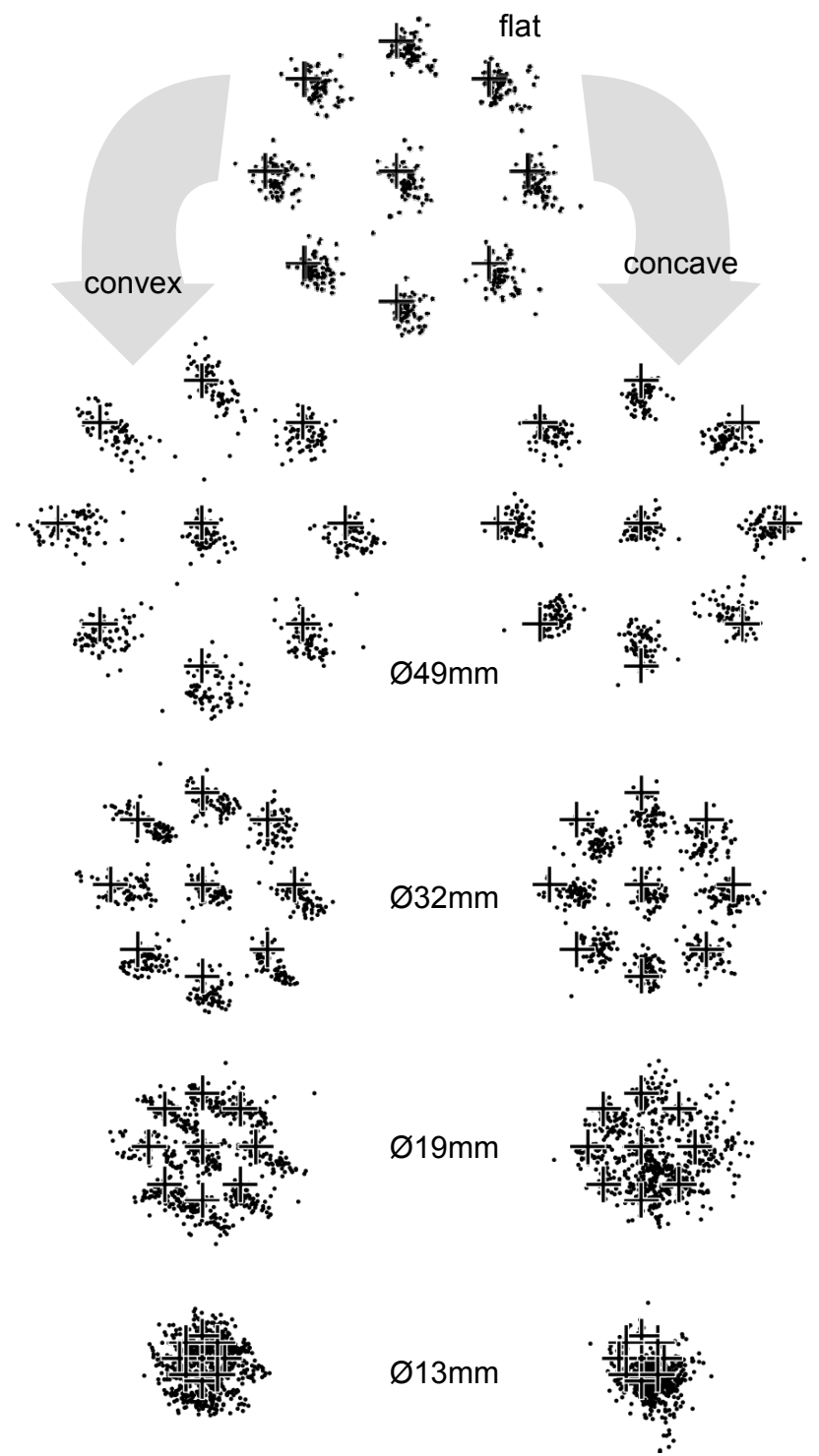

Figure 13: Raw data: all touch locations of all participants by curvature and target orientation/target.

HO: Offsets are oriented along the user's finger

All contact points of all targets combined showed an offset of $1.9 \mathrm{~mm}$. Its direction matched the direction of participant's fingers closely, i.e., it was off by only $5.1^{\circ}$ clockwise from the $45^{\circ}$ participant finger angle. This suggests that offsets are most likely the result of finger direction, rather than, say, head position, which should have produced a north/south oriented offset. This observation matches finger direction offsets previously observed on flat surfaces [13] and supports our hypothesis H0.

The overall effect shows reasonably clearly in Figure 13, where most contact point clusters are offset to the bottom right with respect to their target. An exception is the concave $\varnothing 49 \mathrm{~mm}$ shape. Unlike any of the other curvatures, it showed virtually no global offset, but target-specific offsets towards the center. We discuss this effect in more detail below, and investigated it in a brief follow-up study, also presented in this paper.

\section{H1: Concavity increases offsets, convexity reduces it}

A one way ANOVA found a main effect of curvature on offset $\left(F_{8,88}=5.24, p<.0001\right)$. Post-hoc comparison tests (using a Tukey's HSD test) indicated the significant differences shown in Figure 14a.
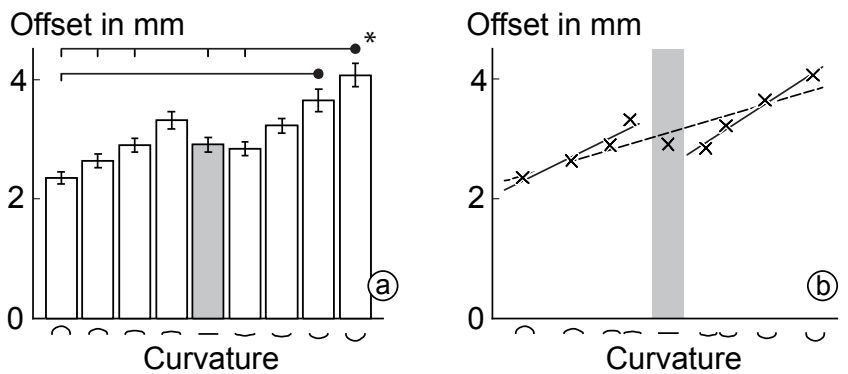

Figure 14: (a) Error offset by surface curvature (+/- 95\% confidence, * significantly different) (b) Linear regression. The $x$-axis is curvature measured as $1 /$ radius.

To understand this relationship better we performed linear regressions (Figure 14b). A single linear regression explains a significant portion of offset $\left(r^{2}=.7768, F_{1,7}=24.36\right.$, $p<.001)$ for the length of the projection vector: $\mathrm{L}=-.45 \times$ curvature +3.1 . Performing regression separately for convex and concave obtains a better fit: for convex $\left(r^{2}=.9187\right.$, $F_{1,2}=22.61, p<.04, \mathrm{~L}=-.76 \times$ curvature +3.5$)$ and for concave $\left(r^{2}=.9752, F_{1,2}=78.77, p<.01, \mathrm{~L}=\right.$ - curvature +2.53$)$.

These results support our hypothesis H1, i.e., error offsets indeed decreased with convexity and increased with concavity, as suggested by their differences in contact area (Figure 4). These findings integrate nicely with the related work on flat surfaces [13], while generalizing from flat to curved surfaces.

\section{H2: Concavity increases spread, convexity reduces it}

Figure 15 illustrates spread across curvatures. Note that we computed spread on a per-user basis. Intuitively, this means that each bar indicates how closely the contact points of a single user are collocated for a target on the respective curvature. For a device to exploit this it needs to employ a per-user calibration, as suggested by [13].

A two way ANOVA found a main effect of curvature on spread $\left(\mathrm{F}_{8,88}=7.62, p<.0001\right)$. Post-hoc multiple means comparison tests found the significant differences shown in Figure 15. 


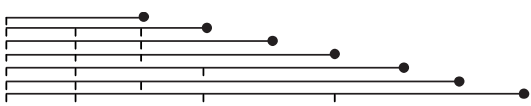

Minimum button size in $\mathrm{mm}$

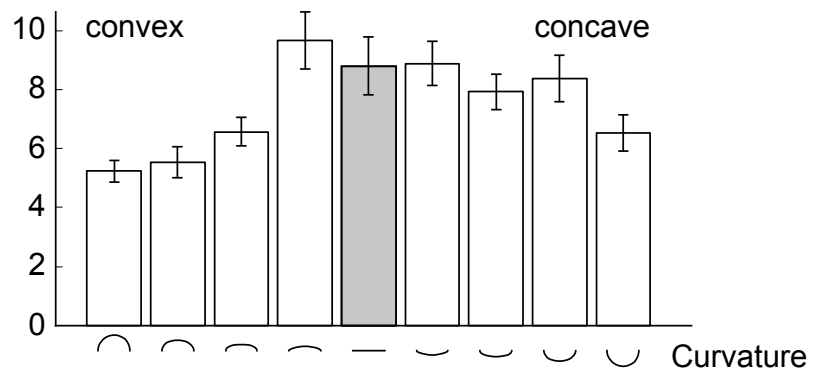

Figure 15: Spread in minimum button size by curvature ( $+/-95 \%$ confidence).

The left half of Figure 15, i.e., the one depicting accuracy on convex shapes, is in general support of our hypothesis $\mathrm{H} 2$ : spread indeed decreased with increasing convexity. The concave shapes, in contrast, came out unexpectedly. Rather than spread increasing further with concavity, spread eventually even decreased.

Figure 16 suggests one possible explanation for this effect. First, a tight cavity confines the finger, which provides users with tactile feedback that can help adjust the position of their finger. Second, the stronger the curvature, the shorter the part of the bowl that is actually concave. The space around the bowl has to be convex in order to connect the concave bowl to the rest of the surface. For very strong curvatures, users' fingers fill out the concave part, so that variations in finger posture lead to changes in the contact area on the convex part, where it leads to smaller changes in contact area, thus reduced spread.

This effect is inherent to the nature of curved surfaces: unlike flat surfaces, curved surfaces are finite and the stronger the curvature, the smaller the surface. This holds for concave, as well as convex, as illustrated also by the FlyEye shown in Figure 2.

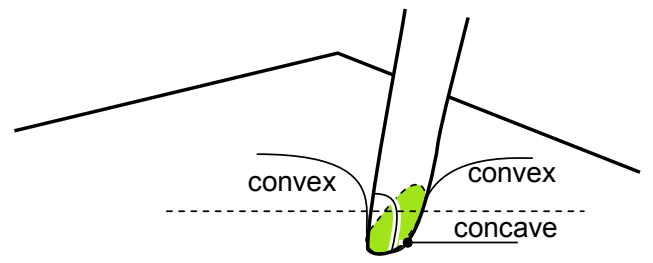

Figure 16: Strongly concave surfaces lead to lower spread. One possible explanation is that they provide tactile guidance. Another one is that they are framed by convex areas, where posture variations cause little variation in contact area.

\section{Q1: Effect of uphill/downhill slope on offset}

As illustrated by Figure 19, participants acquired different targets using different finger postures. For targets located on downhill slopes, participants were more likely to employ a hooked finger posture, while they were more likely to use a straight finger to acquire targets located on uphill slopes. When analyzing slope, we grouped target locations that resulted in similar finger postures, as shown in Figure 17.
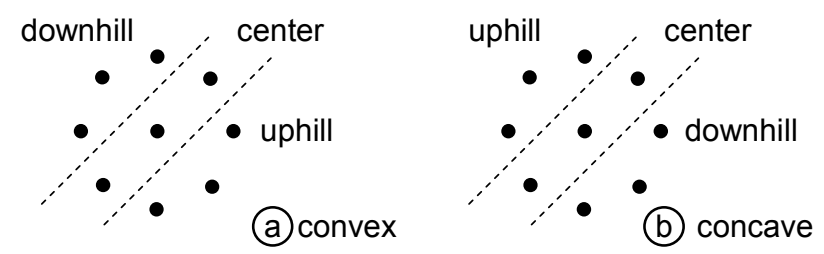

Figure 17: Targets were grouped by slope

A two way ANOVA found a main effect of curvature $\left(\mathrm{F}_{8,88}=5.25, p<.0001\right)$, slope $\left(\mathrm{F}_{2,22}=10.60, p<.001\right)$ and the interaction curvature $\times$ slope $\left(\mathrm{F}_{16,176}=7.02, p<.0001\right)$ on offset. Figure 18 shows pair wise differences.

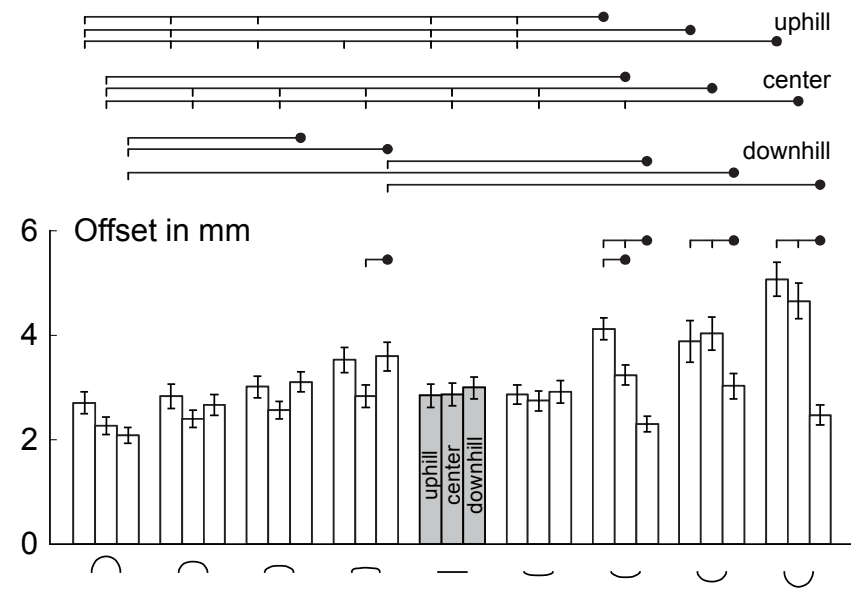

Figure 18: Error offset by curvature and slope: uphill, center, downhill ( $+/-95 \%$ confidence)

Post-hoc multiple means comparison tests (using a Tukey's HSD test) found that the offsets of downhill slopes were generally smaller than the offsets of center slopes, which in turn were smaller than the offsets of uphill slopes. As apparent also in Figure 18, however, this effect was entirely caused by the concave surfaces.
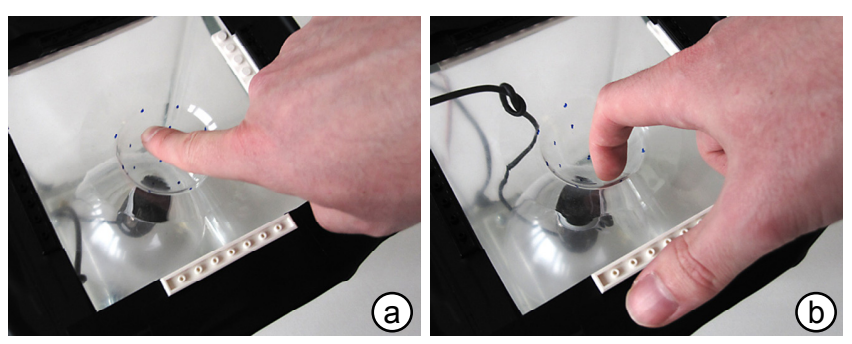

Figure 19: (a) When targeting with a straight finger, the soft bottom side of the user's finger is occluded. (b) Users can see it when targeting with a hooked finger.

One possible explanation for the smaller offsets on downhill slopes is differences in finger posture, i.e., that a hooked finger leads to smaller offsets than a straight finger. On flat surfaces, touch inaccuracy has been attributed to users' inability to monitor the soft fleshy bottom of the finger [26, 14]. As illustrated by Figure 19, users pointing using a hooked finger can see that bottom side. The now invisible side, i.e., the fingernail, is less malleable and thus suffers from the fat finger problem to a much lesser extent. 


\section{Q2: Effect of uphill downhill slope on spread}

A two-way ANOVA found no significant effects of slope on spread.

\section{Summary}

In summary, by adding curvature as a factor, we have generalized the concept of error offsets from flat surfaces [13] to curved surfaces. We found that error offsets depend on curvature (H1) and slope (Q1). Both factors influence error offset, but there is an interaction and most of the error offset of concave targets comes from the uphill slope, where users are forced to target using a straight finger.

The effects of curvature on spread, in contrast, are moderate. There appears to be an effect for convex targets. For concave targets in contrast, multiple confounding factors compensate for each other.

\section{FOLLOW-UP STUDY ON CONCAVE}

As discussed earlier, we speculated that the smallness of the error offsets of the concave targets was caused by participants targeting using a hooked finger. To verify this assumption, we conducted an informal follow-up study with a small number of additional participants. Participants again repeatedly acquired crosshair targets of different orientations. Our main hypothesis was that the lack of offsets on concave surfaces was an artifact of participants employing different finger postures. To investigate this, we varied finger posture this time.

\section{Interface \& Apparatus \& Task}

We used the same curve touch device, screen, button, and footswitch setup as in the main user study (Figure 11). We limited the study to the $\varnothing 49 \mathrm{~mm}$ concave surface, which had displayed the pattern most clearly. Participants performed the same task as before.

To obtain additional data for the slope variable, we broke the single target ring at $45^{\circ}$ zenith angle from the previous study down into an inner ring at $30^{\circ}$ and an outer ring at $60^{\circ}$ zenith angle for an overall 8 outer +8 inner +1 apex $=$ 17 targets.

\section{Additional independent variable: Finger posture}

(1) In the fingertip condition, participants were instructed to acquire all targets using their fingertip. This caused them to acquire targets using a straight finger for the uphill targets and to hook their finger for the downhill targets. (2) In the flat finger condition, they acquired all targets as with a flat finger. This forced them to use a straight finger also for downhill targets. (3) In the free condition participants acquired each target as they chose to-which corresponded to the first study. In order not to influence participants in the free condition, we ran the free condition first. We then counterbalanced the following fingertip and flat finger conditions.

\section{Experimental design}

The study used a $3 \times 17$ within-participant design, with independent variables finger posture (fingertip, flat finger, free) and slope (17 targets organized in two rings plus apex). Participants performed six trials for each finger posture and slope. The order of target orientations was randomized. Each participant completed all conditions, i.e., 3 finger postures $\times 17$ target orientations $\times 6$ trials $=306$ trials per participant.

Participants performed 5 minutes of training before the experiment. They were allowed to take breaks every 34 selections. They all completed the study in 30 minutes or less.

\section{Participants}

We recruited 6 new right-handed participants (2 female) from our institution. They were between 24 and 30 years old. They again received a small compensation for their time and we awarded $€ 20$ to the most accurate participant.

\section{Hypotheses}

Our main hypothesis was that the unexpected lack of a finger direction offset of the $\varnothing 49 \mathrm{~mm}$ surface would only happen in the free and fingertip conditions, but that the flat finger condition would still be subject to the offset.

H0: Offsets are oriented along the user's finger

\section{Results}

Figure 20 and Figure 21 show the raw data, i.e., all contact points obtained during the study by fingertip, flat finger, and free conditions.

As in the main study, the overall error offset of the free condition pointed towards the center rather than in the direction of the finger (the component of the error offset across all targets/slopes pointing in the direction of the finger measured only $0.8 \mathrm{~mm}$ ). This held across all targets including the additional ring of outer targets. For the fingertip condition the finger direction offset was equally small $(0.9 \mathrm{~mm})$. In the flat finger condition, however, we did see a major offset in the direction of the finger $(2.4 \mathrm{~mm})$.
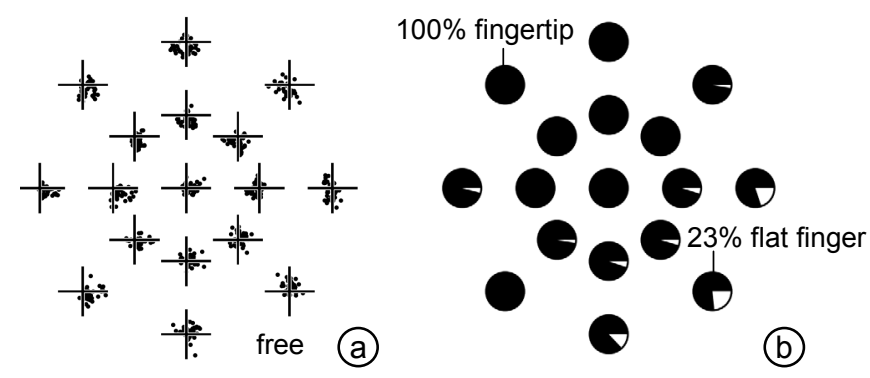

Figure 20: (a) Contact points in the free condition and (b) how much the free condition resembled the fingertip condition.

We reconstructed participants' finger posture in the free condition by comparing the contact area sizes for each touch with the fingertip and flat finger conditions. Figure $20 \mathrm{~b}$ illustrates that participants effectively targeted with the fingertip throughout; a slight tendency towards using a flat finger in the bottom right was weak enough that it had little effect on the error offsets. 

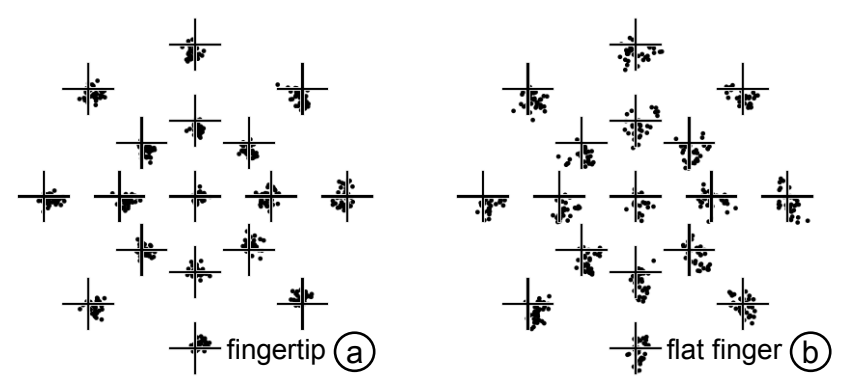

Figure 21: Contact points of (a) the fingertip and (b) the flat finger conditions.

\section{Discussion}

The results of this informal study indicate that the error offsets in the concave condition of the main study are indeed an artifact of finger posture. By switching between a straight and hooked finger, participants always targeted using the finger tip, thereby targeting more accurately in the sense that offsets were reduced.

What remains is the question why the more strongly curved concave conditions seemed to benefit less from the hooked finger posture (see also Figure 13). Figure 22 attempts to explain this by illustrating an effect we observed during piloting For strongly concave surfaces, a hooked finger posture introduces additional targeting errors when it accidentally touches the opposite side of the bowl (Figure 22a). Most pilot users avoided the issue by switching back to a straight finger posture. This avoids the accidental touches, at the expense of reintroducing the increased offset error of the straight finger.
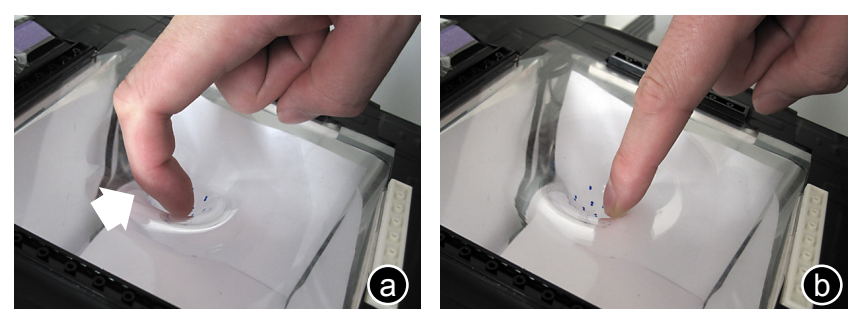

Figure 22: (a) A hooked finger accidentally touching the opposite side of the bowl. (b) Switching back to a straight finger posture avoids the issue at the expense of the increased error.

\section{IMPLICATIONS FOR DESIGN}

On the one hand, the findings presented in this paper are intended to deepen our understanding of targeting on nonplanar surfaces. On the other hand, the same findings can be used to inform the design of curved touch devices as well as the design of interfaces running on such devices.

For device designers, we found two additional factors influencing error offsets, namely surface curvature and slope. Knowledge of these offsets allows engineers to compensate for these effects by coding corrective offsets into their device drivers, which will increase device accuracy (as demonstrated for flat surfaces by [13]).

For interface designers, minimum button sizes are relevant, because they help design usable interfaces. Our findings suggest placing targets on points of extreme curvatures in order to make them easier to acquire, so application designers might want to use them for frequently used functions.

\section{CONCLUSIONS}

Our findings indicate that the curvature of touch surfaces impacts targeting in terms of spread/minimum button size and in particular in terms of systematic offsets. This information can help designers of curved touch devices improve their devices.

As future work, we plan to study the impact of shape on more complex interactions, such as grasping. New findings in this space may one day provide the missing link between HCI and the disciplines that have discussed form factor and shape all along, such as industrial design.

\section{ACKNOWLEDGMENTS}

We thank Sean Gustafson and Christian Holz for valuable comments and help on statistics, and all participants of our studies for their time.

\section{REFERENCES}

1. Beaudouin-Lafon, M. and Mackay, W. E. Reification, polymorphism and reuse: three principles for designing visual interfaces. In Proc. AVI'00, 102-109.

2. Benko, H., Wilson, A. D. and Baudisch, P. Precise selection techniques for multi-touch screens. Proc. CHI'06, 1263-1272.

3. Benko, H., Wilson, A. D., and Balakrishnan, R. Sphere: multi-touch interactions on a spherical display. In Proc. UIST'08. 77-86.

4. Cao, X., Wilson, A. D., Balakrishnan, R., Hinckley, K. and Hudson, S. E. Shapetouch: leveraging contact shape on interactive surfaces. In Proc. Tabletop'08, 129-136.

5. Chapanis, A. Theory and methods for analyzing errors in man-machine systems. Annals of the New York Academy of Sciences 51, Human Engineering (1951), 11791203.

6. Fitts, P.M. 1954. The information capacity of the human motor system in controlling the amplitude of movement. Journal of Experimental Psychology 47, 381-391.

7. Forlines, C., Wigdor, D., Shen, C. and Balakrishnan, R. Direct-touch vs. mouse input for tabletop displays. In Proc. CHI'07, 647-656.

8. Grossman, T. and Balakrishnan, R. A probabilistic approach to modeling two-dimensional pointing. TOCHI 12, 3 (Sep. 2005), 435-459.

9. Han, J. Low-cost multi-touch sensing through frustrated total internal reflection. In Proc. UIST'05, 115-118.

10. Harrison, C. and Hudson, S. Providing dynamically changeable physical buttons on a visual display. In Proc. CHI'09, 299-308.

11. Harrison, C., Tan, D. and Morris, D. Skinput: appropriating the body as an input surface. In Proc. CHI '10, 453-462. 
12. Holman, D., Vertegaal, R., Altosaar, M., Troje, N. and Johns, D. 2005. Paper windows: interaction techniques for digital paper. In Proc. CHI '05, 591-599.

13. Holz, C. and Baudisch, P. The generalized perceived input point model and how to double touch accuracy by extracting fingerprints. In Proc. CHI'10, 581-590.

14. Holz, C. and Baudisch, P. Understanding touch. To appear in Proc. CHI'11, 10 pages.

15. Moscovich, T. Contact area interaction with sliding widgets. In Proc. UIST'09, 13-22.

16. Potter, R., Weldon, L. and Shneiderman, B. Improving the accuracy of touch screens: an experimental evaluation of three strategies. In Proc. CHI'88, 27-32.

17. Rekimoto, J. SmartSkin: an infrastructure for freehand manipulation on interactive surfaces. In Proc. CHI '02, 113-120.

18. Rosenberg, I. and Perlin, K. The UnMousePad: an interpolating multi-touch force-sensing input pad. In Proc. SIGGRAPH'09, 1-9.

19. Sato, T., Mamiya, H., Koike, H. and Fukuchi, K. PhotoelasticTouch: transparent rubbery tangible interface using an LCD and photoelasticity. In Proc. UIST'09, 43-50.

20. Schwesig, C., I. Poupyrev, and E. Mori. Gummi: a bendable computer. In Proc. of $\mathrm{CHI}^{\prime} 04$, pp. 263-270.

21. Sears, A. and Shneiderman, B. High precision touchscreens: design strategies and comparisons with a mouse. Int. J. Man-Mach. Stud. 34, 4 (Apr. 1991), 593613.

22. Siek, K.A., Rogers, Y. and Connelly, K.H. Fat finger worries: how older and younger users physically interact with PDAs. In Proc. INTERACT'05, 267-280.

23. Taylor, B. T. and Bove, V. M. The bar of soap: a grasp recognition system implemented in a multi-functional handheld device. In CHI'08 Extended Abstracts, 34593464.

24. Villar, N., Izadi, S., Rosenfeld, D., Benko, H., Helmes, J., Westhues, J., Hodges, S., Ofek, E., Butler, A., Cao, $\mathrm{X}$. and Chen, B. Mouse 2.0: multi-touch meets the mouse. In Proc. UIST '09, 33-42.

25. Vlack, K., Mizota, T., Kawakami, N., Kamiyama, K., Kajimoto, H. and Tachi, S. GelForce: a vision-based traction field computer interface. In $\mathrm{CHI}^{\prime} 05$ Extended Abstracts, 1154-1155.

26. Vogel, D. and Baudisch, P. Shift: a technique for operating pen-based interfaces using touch. In Proc.CHI'07, 657-666.

27. Wang, F. and Ren, X. 2009. Empirical evaluation for finger input properties in multi-touch interaction. In Proc. CHI'09. 1063-1072.

28. Wigdor, D., Forlines, C., Baudisch, P., Barnwell, J. and Shen, C. LucidTouch: a see-through mobile device. In Proc. UIST'07, 269-278.

29. Wimmer, R. FlyEye: grasp-sensitive surfaces using optical fiber. In Proc. TEI '10, 245-248. 